\title{
HtrA2 enhances the apoptotic functions of p73 on bax
}

\author{
M Marabese ${ }^{\star}$, M Mazzoletti, F Vikhanskaya and M Broggini
}

Regulation of the $\mathrm{p} 73$ gene is complex due to the presence of two promoters and the very complex mRNA maturation in both the $\mathrm{N}$-terminal and $\mathrm{C}$-terminal parts of the protein. We have found an additional regulation mechanism for the $\mathrm{p} 73-\alpha$ form that occurs through proteolytic cleavage connected to the activity of the serine protease HtrA2. Following apoptotic stimuli, HtrA2 accumulates in the nucleus and cleaves $p 73 \alpha$ in the C-terminal portion, enabling the protein to increase its transactivation activity on the apoptotic gene bax but not on the cell-cycle regulator gene p21. In the presence of HtrA2, p73 is more prone to cause caspase activation and nuclei fragmentation: p73 needs HtrA2 to activate and enhance its apoptotic functions. This new relation between $\mathrm{p73}$ and HtrA2 may help to understand the different behavior of the p73 protein in cell physiology and in the responses of cancer cells to chemotherapy.

Cell Death and Differentiation (2008) 15, 849-858; doi:10.1038/cdd.2008.7; published online 8 February 2008

p73 is a transcription factor belonging to the p53 family, together with $\mathrm{p} 63 .^{1,2}$ It shares a high degree of sequence homology with p53 and can bind to DNA in a sequencespecific manner in the promoters presenting p53-responsive elements, activating the transcription of p53 target genes involved in cell-cycle arrest and apoptosis. ${ }^{3-6}$ All family members have three protein domains: an N-terminal transactivation domain, a central DNA-binding domain (DBD) and a C-terminal oligomerization domain. ${ }^{2}$ The $5^{\prime}$ portion of the gene has two transcription initiation sites that can generate transactivation-proficient (TA) proteins or proteins lacking the TA domain but containing an extra exon (exon $3^{\prime}$ ) not present in full-length proteins: $\mathrm{N}$-terminus deficient (DN) isoforms. ${ }^{7-9}$ The TAp73 isoforms exert transcriptional activity, whereas the DNp73 variants, lacking the transactivation domain, cannot directly induce gene expression and do not induce apoptosis or cell-cycle arrest. ${ }^{10}$ The main role of $\mathrm{N}$-truncated isoforms is regulatory activity, since they exert a dominant-negative effect on p53 and TAp73 by blocking their transactivating activity to induce cell growth arrest or cell death. ${ }^{11,12}$ However, most of the changes in the p73 gene generated by alternative splicing occurs at the C-terminus. There are at least six different isoforms, from $\alpha$ to $\eta .^{5,13}$ The C-terminus of the $\alpha$-isoform of p63 and p73 contains the sterile $\alpha$-motif domain. ${ }^{2}$ The relative amounts of p53, TAp73 and DNp73 are thought to be involved in determining cell death and cell-cycle arrest or cell proliferation. ${ }^{14,15}$ The p73 steadystate protein levels seem to define the role of $p 73$ protein members in tumorigenesis and it would therefore be very useful to identify proteins to control their steady-state levels.

High temperature requirement A (HtrA2) is a mammalian serine protease that resides in the mitochondria of healthy cells, but apoptotic stimuli cause it to be released into the cytoplasm, where it binds the inhibitor of apoptotic proteins (IAPs). ${ }^{16-18}$ This results in proteolytic degradation of these inhibitor proteins and in the following activation of the caspase cascade mechanism. HtrA2 is also involved in caspaseindependent cell death through its serine protease activity exerted directly on specific targets. ${ }^{16-18}$

There is evidence linking HtrA2 to p53. In particular, HtrA2 may help in determine some of the proapoptotic functions of $\mathrm{p} 53$. The proposed mechanism passes through a p53mediated increase in HtrA2 transcriptional levels, which would increase the degradation of IAPs. ${ }^{19}$

Here we provide evidence that HtrA2 is also linked to p73. However, p73 does not directly activate HtrA2 transcription. Rather, HtrA2, through its serine protease activity, can cleave p73 in the C-terminus, leading to a truncated protein with increased apoptotic function. This suggests a new mechanism of activation of p73 apoptotic functions.

\section{Results}

HtrA2 cleaves p73 in vitro. Starting from data on $p 53,{ }^{19}$ we verified whether p73 could also induce HtrA2 mRNA expression after transfection. As shown in Figure 1a, neither TA nor DN p73 isoforms promoted HtrA2 mRNA expression in H1299 non-small-cell lung carcinoma cells. In the same experimental conditions, p53 also had no effect on HtrA2 expression. As a control, we verified the induction of p21 mRNA after transfection (Figure 1a) and the effective expression of transfected p53 and p73 isoforms (Figure 1b). Transfection of $\mathrm{TAp} 73 \alpha$, p53 or DNp73 $\alpha$ did not enhance HtrA2 protein levels in these cells (Figure 1c). Furthermore, the p53 downregulation by siRNA failed to modulate HtrA2 expression in both untreated and doxorubicin-treated conditions (Figure 1d). In these experiments, however, expression of transcription-proficient p73 isoforms promoted the release of $\mathrm{HtrA} 2$ from the mitochondria to the

Laboratory of Molecular Pharmacology, Department of Oncology, Istituto di Ricerche Farmacologiche, 'Mario Negri', via La Masa 19, Milan 20156, Italy

*Corresponding author: M Marabese, Laboratory of Molecular Pharmacology, Department of Oncology, Istituto di Ricerche Farmacologiche 'Mario Negri', via La Masa 19, Milan 20156, Italy. Tel: + 023901 4236; Fax: + 39023901 4734; E-mail: marabese @marionegri.it

Keywords: p73; HtrA2/Omi; apoptosis; cleavage; bax

Abbreviations: TA, transactivation proficient; DN, N-terminus deficient; HtrA2, high temperature requirement A; MEF, mouse embryonic fibroblast; P1, TA promoter; P2, DN promoter; doxo, doxorubicin

Received 20.12.07; accepted 04.1.07; Edited by KH Vousden; published online 08.2.08 


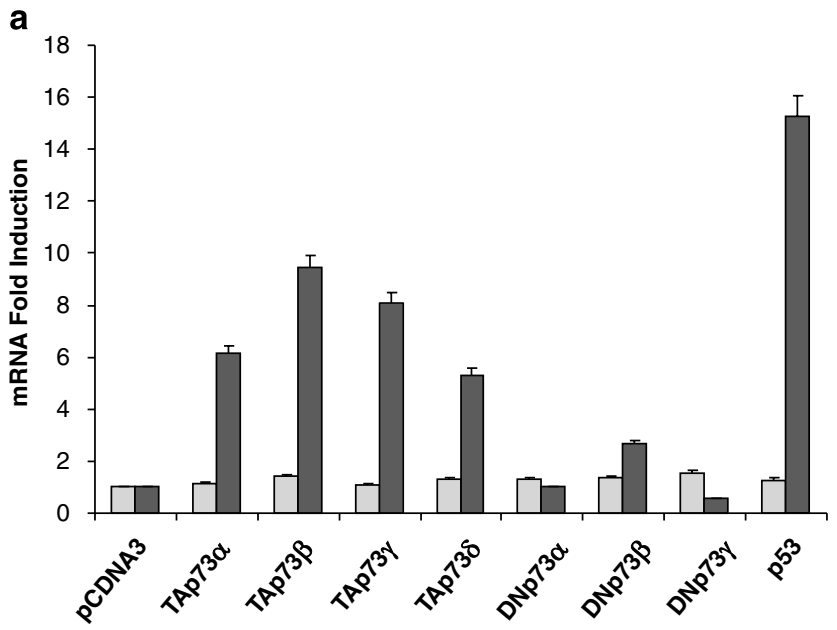

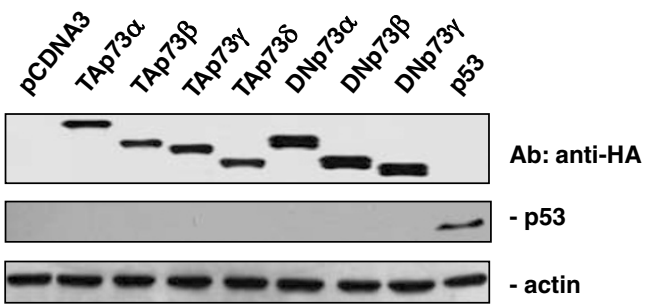

C

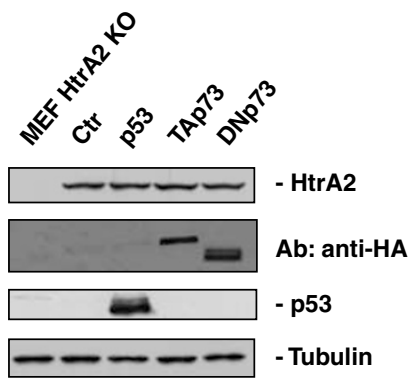

d

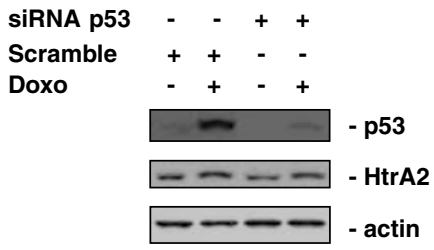

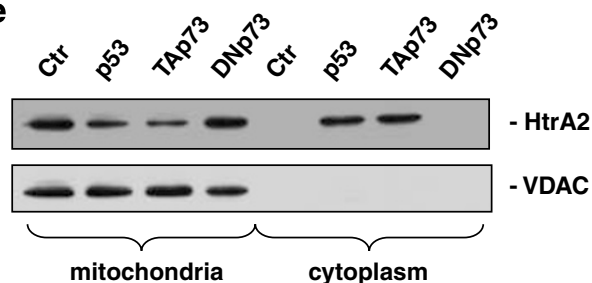

f

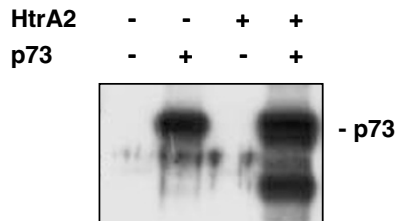

Figure 1 (a) Expression of exogenous p73 isoforms or p53 does not influence the transcriptional level of HtrA2. H1299 cells seeded in six-well plates were transfected with equal amounts $(2 \mu \mathrm{g})$ of plasmids encoding for different isoforms of p73, p53 and pCDNA3 as control. Twenty-four hours after transfection, total RNA was extracted and analyzed by real-time RT-PCR to quantify the expression of HtrA2 (gray bars) and p21 (black bars). The average of three different experiments \pm S.D. is shown. (b) Expression of p73 isoforms and p53. Aliquots of cells used in the previous experiment were used to analyze by western blot the expression of the all transfected proteins. Twenty micrograms of each sample were resolved on SDS-PAGE gel, followed by immunoblotting. Actin was used as internal loading control. (c) HtrA2 protein levels are not influenced by ectopically expressed p53 or p73 isoforms. $\mathrm{H} 1299$ cells seeded in six-well plates were transfected with equal amounts $(2 \mu \mathrm{g})$ of plasmid encoding for p53 or p73 isoforms. Twenty-four hours after transfection, $20 \mu \mathrm{g}$ of whole-cell lysates were resolved on a SDS-PAGE gel, followed by immunoblotting. Proteins extracted from HtrA2knockout MEFs (column 1) was used as negative HtrA2 control to confirm antibody specificity. Tubulin was used as internal loading control. (d) Downregulation of p53 by siRNA does not influence HtrA2 levels: HCT116 cells seeded in 24-well plates were transfected with equal amounts (35 pmoles) of scramble or p53 siRNA. Forty-eight hours after transfection, $20 \mu \mathrm{g}$ of whole-cell lysates were resolved on a SDS-PAGE gel, followed by immunoblotting. Actin was used as internal loading control. (e) Expression of exogenous p53 or p73 isoforms influences the localization of HtrA2. H1299 cells seeded in 10-cm plates were transfected with $4 \mu \mathrm{g}$ plasmid encoding for p53 or p73 isoforms. Twenty-four hours after transfection, samples were subjected to sub-cellular fractionation to analyze the protein of interest in each compartment. Twenty micrograms of each sample were resolved on SDS-PAGE gel, followed by immunoblotting. VDAC was used as marker of mitochondria. (f) Coexpression of p73 and HtrA2 results in the appearance of new band at lower molecular weight. $\mathrm{H} 1299$ cells seeded in six-well plates were cotransfected with $2 \mu \mathrm{g}$ plasmid encoding for p73 and $2 \mu \mathrm{g}$ plasmid encoding for HtrA2. Twenty-four hours after transfection, $20 \mu \mathrm{g}$ of whole-cell lysates were resolved on a SDS-PAGE gel, followed by immunoblotting for p73

cytoplasm (Figure 1e). Coexpression of p73 and HtrA2 resulted in the appearance of new bands at lower molecular weight (Figure 1f). This prompted us to further analyze the interaction between p73 and HtrA2, to see whether this serine protease could directly cleave p73 isoforms. We synthesized six radiolabelled $\left[{ }^{35} \mathrm{~S}\right]-\mathrm{p} 73$ isoforms, including four C-terminal TA one (TAp73 $\alpha$, TAp73 $\beta$, TAp73 $\gamma$ and TAp73 $\delta$ ) and two C-terminal DN isoforms (DNp73 $\alpha$ and DNp73 $\beta$ ). Only TAp73 $\alpha$ (Figure 2a) and DNp73 $\alpha$ (Figure 2b) were cleaved when incubated with $\mathrm{HtrA} 2$ in vitro.

In vitro synthesized radiolabelled $\left[{ }^{35} \mathrm{~S}\right]-\mathrm{TAp} 73 \alpha$ was incubated with increasing amounts of wild-type HtrA2 or a proteolysis-defective mutant, S306A HtrA2. As shown in Figure 2c, only wild-type HtrA2 cleaved TAp73 $\alpha$. This effect was specific for HtrA2 since it was not seen with the proteolysis-defective mutant. These results indicate that the part of protein present only in the $\alpha$-isoforms is likely to contain an amino-acid sequence recognized by $\mathrm{HtrA} 2$.

To confirm the potential cleavable site in a specific domain of the p73 protein, we incubated different in vitro synthesized radiolabelled $\left[{ }^{35} \mathrm{~S}\right]-\mathrm{p} 73$ domains with recombinant $\mathrm{HtrA} 2$, using the $\mathrm{N}$-terminal domain (NTD), the DBD and the C-terminal domain (CTD) as illustrated in Figure 2d. In agreement with previous experiments, only the CTD was cleaved when incubated with recombinant HtrA2 (Figure 2g). The other domains (NTD and DBD) were not cleaved when incubated with the serine protease under the same experimental conditions (Figure $2 \mathrm{e}$ and $\mathrm{f}$ ).

HtrA2 cleaves p73 in cells. To investigate whether p73 was also cleaved in cells, we cotransfected TAp73 $\alpha$ and HtrA2 in the $\mathrm{H} 1299$ cell line. As shown in Figure 3a, lane 5, HtrA2 
a

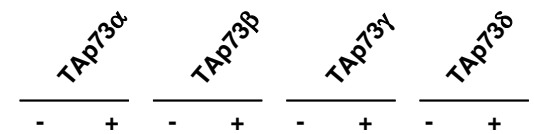

HtrA2

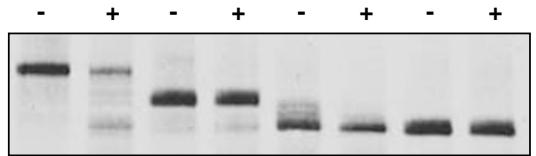

b

HtrA2

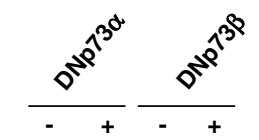

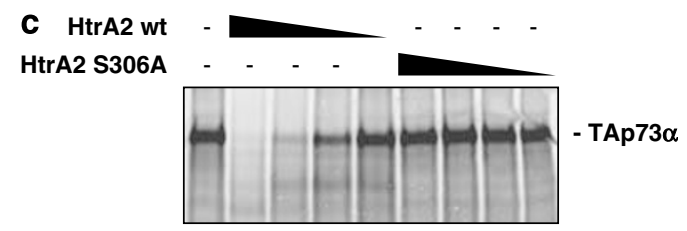

e HtrA

f HtrA2

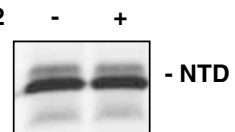

d

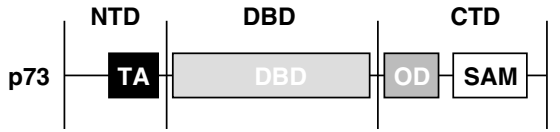

g HtrA

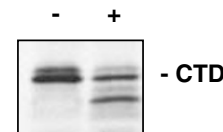

Figure $2 \mathrm{HtrA} 2$ exerts its protease activity only on certain $\mathrm{p} 73$ isoforms. (a) In vitro translated radiolabelled $\left.{ }^{35} \mathrm{~S}\right]-\mathrm{TAp} 73$ isoforms $(\alpha, \beta, \gamma$ and $\delta)$ were incubated for $2 \mathrm{~h}$ at $37^{\circ} \mathrm{C}$ with or without recombinant wild-type HtrA2 at the concentration of $0.42 \mu \mathrm{M}$. Samples were resolved on a SDS-PAGE gel, followed by autoradiography. (b) The same experiment as in panel (a) was repeated using DNp73 $\left(\alpha\right.$ and $\beta$ ) isoforms. (c) The in vitro translated radiolabelled $\left[{ }^{35} \mathrm{~S}\right.$ ]-TAp73 $\alpha$ isoform was incubated with increasing amounts of wild-type or mutant S306A HtrA2 in the conditions described in panel (a). (d) Schematic representation of p73 domains. (e-g) Plasmids encoding for p73 domains were in vitro translated with radiolabelled $\left[{ }^{35} \mathrm{~S}\right]$ methionine to find the cleavage region targeted by HtrA2 on p73. The recombinant NTD (e), the DBD (f) and the CTD (g) of p73 were produced and incubated for $2 \mathrm{~h}$ at $37^{\circ} \mathrm{C}$ with or without recombinant wild-type $\mathrm{HtrA2}$ at the concentration of $0.42 \mu \mathrm{M}$

cleaved TAp73 $\alpha$ in cells. However, in accordance to the in vitro data, catalysis-defective $\mathrm{HtrA} 2$ was unable to induce TAp73 $\alpha$ fragmentation (Figure 3a, lane 6). The next step was to confirm that HtrA2 could cleave p73 in the CTD. Figure $3 b$ shows that cotransfection of the different plasmids expressing NTD, DBD and CTD domains with the HtrA2encoding plasmid resulted in the cleavage only of CTD, confirming the in vitro findings. We then investigated whether the S306 HtrA2 mutant could compete for the cleavage site on p73. We cotransfected different ratio of mutant HtrA2/ wild-type $\mathrm{HtrA} 2$ together with a fixed amount of TAp73 $\alpha$. As shown in Figure $3 c$, the mutant clearly competed with the wild-type HtrA2 on the cleavage site on TAp73 $\alpha$ and at the highest doses completely wrong-footed the wild type.

As HtrA2 can bind the IAPs promoting their degradation, we checked whether caspases were directly implicated in p73 cleavage cotransfecting TAp73 $\alpha$ and HtrA2 with or without the pan-caspase inhibitor Z-vad-fmk. Figure 3d shows that Z-vadfmk did not affect HtrA2's activity on p73, ruling out any direct caspase activity on p73 cleavage. The effect of Z-vad-fmk treatment was assessed by measuring caspase- 3 and caspase-7 activity in a fluorimetry-based assay (Figure $3 \mathrm{e}$ ).

HtrA2 and p73 interact in the nucleus. A recent work by Kuninaka et al. ${ }^{20}$ reported that $\mathrm{HtrA} 2$ serine protease can be present in the nucleus. Since p73 is a nuclear transcriptional factor, we tested whether HtrA2 could in fact be present in this compartment in our system too. Figure 4A indicates that in unstressed conditions HtrA2 is not found in the nucleus but, after doxo treatment, it did appear there, in the same compartment as p73. We then checked the presence of HtrA2 in the nucleus by confocal microscopy, confirming its presence only after drug treatment (Figure 4B, slide g). In addition, spots of colocalization between HtrA2 and p73 in the nucleus after doxo treatment were found (Figure 4B, slide $\mathrm{h}$ ). The last results prompted us to further analyze the possible in vivo interaction between p73 and HtrA2 by co-immunoprecipitation experiments. Data presented in Figure $4 \mathrm{C}$ clearly show that $\mathrm{HtrA} 2$ and $\mathrm{p} 73 \alpha$ coimmunoprecipitate in cells when overexpressed while p73 $\beta$ did not. We then checked if $\mathrm{p} 73$ and HtrA2 could interact at endogenous level in the nucleus. Only after doxo treatment, $\mathrm{HtrA} 2$ enters in the nucleus, as demonstrated in confocal experiments (Figure 4B), and co-immunoprecipitates with p73 (Figure 4D).

Endogenous p73 is cleaved by HtrA2 and endogenous HtrA2 cleaves p73. To investigate whether HtrA2 cleaved endogenous p73, we overexpressed wild-type HtrA2 in H1299 cells and analyzed the cleavage of the target protein by western blot. The exogenous HtrA2 led to the appearance of a low-molecular-weight band, indicating that endogenous TAp73 $\alpha$ was effectively cleaved in the presence of active HtrA2 (Figure 4E).

We also tested whether endogenous HtrA2 cleaved synthesized radiolabelled $\left[{ }^{35} \mathrm{~S}\right]-\mathrm{TAp} 73 \alpha$ in vitro, by incubating the radiolabelled $\left[{ }^{35} \mathrm{~S}\right]-\mathrm{TAp} 73 \alpha$ with mitochondria extract from wild-type and HtrA2-knockout mouse embryonic fibroblast (MEFs). Only the incubation of radiolabelled $\left[{ }^{35} \mathrm{~S}\right]-\mathrm{TAp} 73 \alpha$ with wild-type $\mathrm{HtrA2}$ mitochondria resulted in p73 cleavage (compare lanes 2 and 4 of Figure 4F). MEF treatment with doxo $2 \mu \mathrm{M}$ for $24 \mathrm{~h}$ resulted in the release of $\mathrm{HtrA} 2$ (and cytochrome $c$ ) from the mitochondria into the cytosol (Figure 4G). As a consequence of this physiological process, mitochondria extract from treated cells contained less HtrA2 than untreated cells (compare lanes 3 and 4 of Figure 4G). Incubation of radiolabelled $\left.{ }^{35} \mathrm{~S}\right]-\mathrm{TAp} 73 \alpha$ with mitochondria from treated cells resulted in slightly less p73 cleavage than 
a

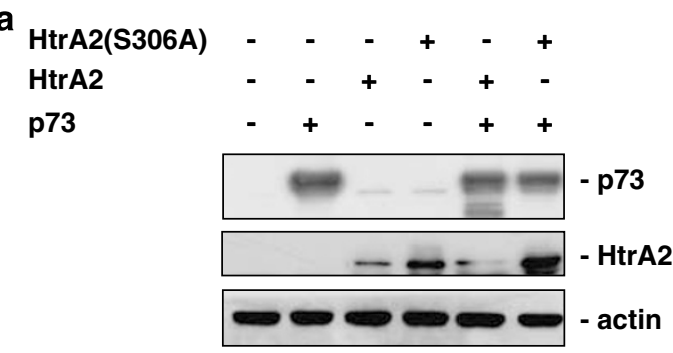

b HtrA2 wt - + -

HtrA2 S306A - $-\quad+$

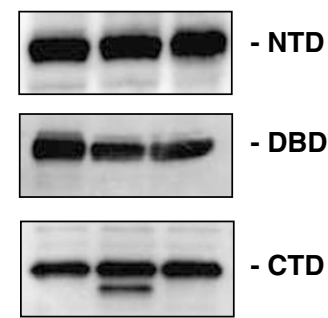

c $\begin{array}{lllllllllllll}\text { p73 } & - & + & - & - & + & + & + & + & + & + & + & + \\ \text { HtrA2 } & - & - & + & - & + & - & + & + & + & + & + & + \\ \text { HtrA2 S306A } & - & - & - & + & - & + & & & & & & \end{array}$

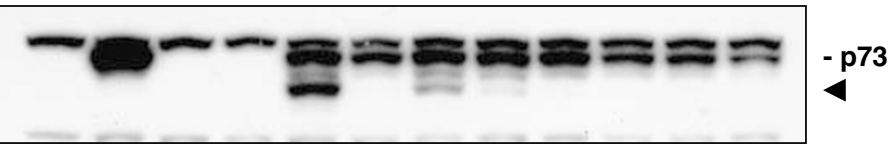

d

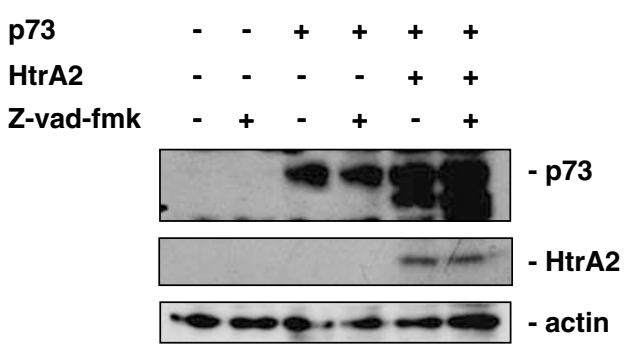

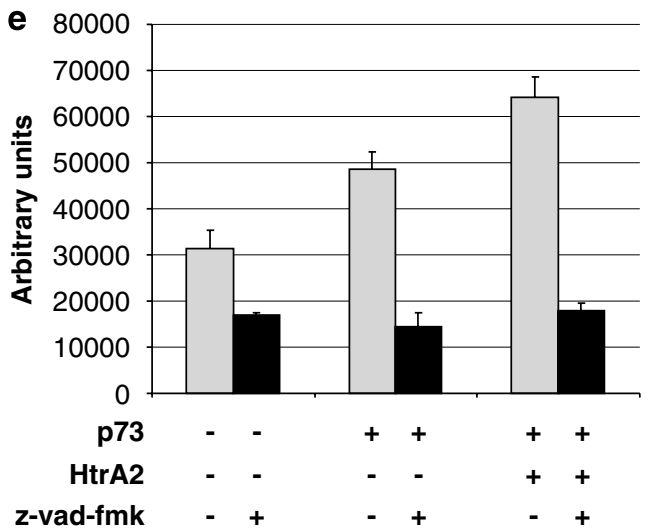

Figure $3 \mathrm{HtrA} 2$ proteolytic activity on p73 in cells. (a) H1299 cells seeded in six-well plates were cotransfected with plasmid encoding for TAp73 $\alpha$ (2 $\mu \mathrm{g}$ ), with wild-type or mutant HtrA2 $(2 \mu \mathrm{g})$. Twenty-four hours after transfection, $20 \mu \mathrm{g}$ of whole-cell lysates were resolved on a SDS-PAGE gel, followed by immunoblotting. Actin was used as internal loading control. (b) Plasmids encoding for p73 domains were cotransfected in $\mathrm{H} 1299$ cells to confirm the cleavage region targeted by HtrA2 on p73. The recombinant NTD, the DBD and the CTD of $p 73$ were cotransfected with wild type or mutant HtrA2. Twenty-four hours after transfection, $20 \mu \mathrm{g}$ of whole-cell lysates were resolved on SDSPAGE gel, followed by immunoblotting. (c) Fixed amounts of TAp73 $\alpha$-encoding plasmids were cotransfected with increasing ratios, between $1: 1$ and $1: 50$, of inactive HtrA2 (S306A)/wild-type HtrA2. Twenty-four hours after transfection, lysates were resolved on SDS-PAGE gel, followed by immunoblotting with an anti-p73 antibody. The intensity of the degraded form depends on the ratio of wild type to mutant HtrA2 isoforms. (d) Caspase activity is not involved in p73 cleavage. H1299 cells were transfected with p73 plasmid, HtrA2 plasmid or both 30 min after treatment with pan-caspase inhibitor. Twenty-four hours after transfection, $20 \mu \mathrm{g}$ of each sample were resolved on SDS-PAGE gel, followed by immunoblotting. Actin was used as internal loading control. (e) Z-vad-fmk inhibits caspase activity: H1299 cells transfected with p73 plasmid, HtrA2 plasmid or both $30 \mathrm{~min}$ after treatment with pan-caspase inhibitor were seeded in 96-well plates. Twenty-four hours after transfection, caspase 3 and caspase 7 activities were assessed. The average of three different replicates \pm S.D. is shown

with mitochondria from untreated cells (compare lanes 3 and 2 of Figure 4F).

Additional proof linking the serine protease HtrA2 and the transcriptional factor p73 comes from the analysis of p73 endogenous basal levels in HtrA2-knockout murine MEFs. In these cells, which have no HtrA2 activity, basal levels of p73 were higher than in wild-type MEFs, clearly suggesting a correlation between $\mathrm{HtrA} 2$ and p73 and, when HtrA2 was present, TAp73 $\alpha$ levels were lower (Figure $4 \mathrm{H}$ ). To verify the presence of a p73 lower-molecular-weight band in wild-type MEFs, we loaded SDS-PAGE with 10 times more proteins and in this forced condition we detected the processed form of TAp73 $\alpha$ (Figure 4I).
HtrA2 enhances the promoter activity of bax. We then investigated the functional role of the cleavage process exerted by HtrA2 on p73 and, in particular, if the cleavage of p73 could result in different regulation on p73-responsive genes. To answer this question, we checked basal promoter activity on four classical p73 downstream genes: the cellcycle regulator p21, the apoptosis inducer bax, the E3ubiquitine ligase $\mathrm{mdm} 2$ and the angiogenic factor vegf. The luciferase assay clearly indicated that the basal activity of bax promoter was lower in HtrA2-knockout MEFs than in HtrA2 wild-type MEFs (Figure 5A). The basal activity of the others promoter, p21, mdm2 and vegf, was comparable in HtrA2 wild-type and HtrA2-knockout MEFs (Figure 5B-D). 

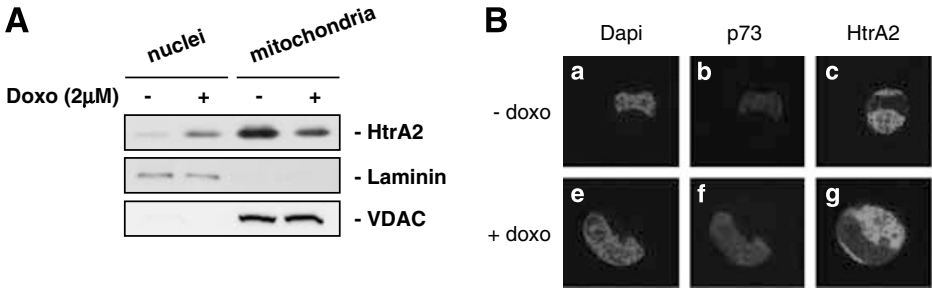

E

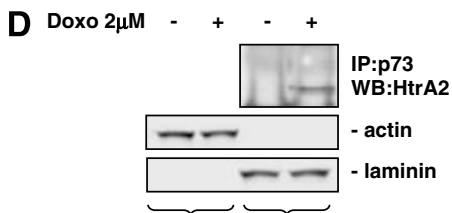

cytoplasm nuclei

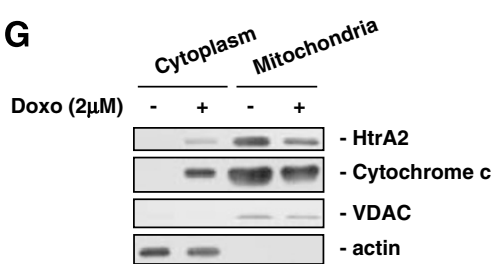

H

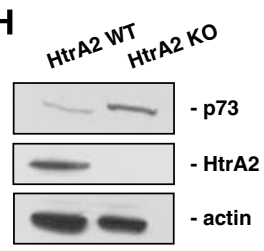

C
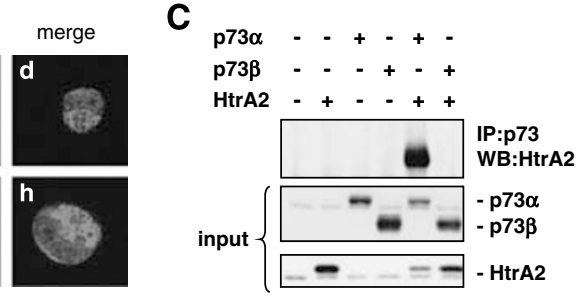

$\mathbf{F}$
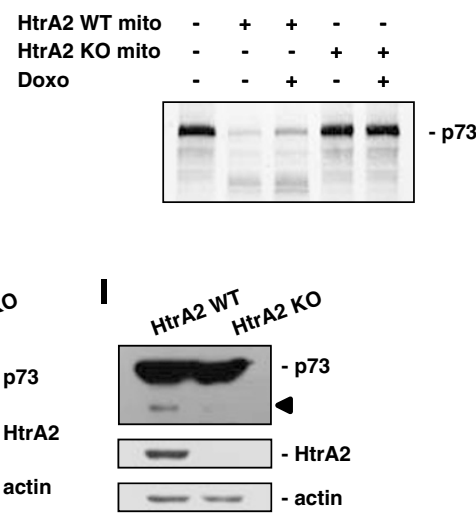

Figure 4 (A) HtrA2 is present in the nucleus after DNA damage. HtrA2 wild-type MEFs were treated with doxo $2 \mu \mathrm{M}$. Twenty-four hours later, samples were subjected to sub-cellular fractionation to analyze the presence of $\mathrm{HtrA2}$ in the nucleus. Twenty micrograms of each sample were resolved on SDS-PAGE gel, followed by immunoblotting. VDAC was used as a mitochondrial marker and laminin as nuclear marker to ensure the absence of cross-contamination. (B) HtrA2 and p73 colocalize in cells: H1299 cells were seeded on a coverslip and twenty-four hours later were treated with doxo $1 \mu \mathrm{M}$ for $24 \mathrm{~h}$. Cells were then stained and analyzed by confocal microscopy. Images are confocal analysis of cells stained with DAPI (a and e), anti-p73 ( $b$ and $f$, red), anti-HtrA2 (c and g, green). The right panels ( $d$ and $h$ ) represent merged images. (C) HtrA2 and p73 $\alpha$ co-immunoprecipitate when overexpressed. $\mathrm{H} 1299$ cells transfected with $\mathrm{p} 73$ plasmid, HtrA2 plasmid or both were lysated and protein extract were immunoprecipitated with anti-p73 antibody. Immunoprecipitates were then resolved on SDS-PAGE gel, followed by HtrA2 immunoblotting. An aliquot of input was also resolved on SDS-PAGE gel as a control. (D) Endogenous HtrA2 and p73 co-immunoprecipitate at endogenous levels after doxo treatment. $\mathrm{H} 1299$ cells were treated overnight with doxo $2 \mu \mathrm{M}$. At the end of the treatment cell fractionation was performed and nuclear extracts were immunoprecipitated with anti-p73 antibody. Immunoprecipitates were then resolved on SDS-PAGE gel, followed by HtrA2 immunoblotting. An aliquot of cytoplasm and nuclear proteins was also resolved on SDS-PAGE gel as a fractionation control. (E) Endogenous HtrA2 acts on p73. H1299 cells were transfected with TAp73 $\alpha$-encoding plasmid. Twenty-four hours after transfection, $150 \mu \mathrm{g}$ of whole-cell lysates were resolved on SDS-PAGE gel, followed by immunoblotting. Actin was used as internal loading control. (F) p73 is cleaved by mitochondrial extract from wild-type HtrA2 MEFs. In vitro translated radiolabelled $\left[{ }^{35} \mathrm{~S}\right]-\mathrm{p} 73$ was incubated for $2 \mathrm{~h}$ at $37^{\circ} \mathrm{C}$ with $10 \mu \mathrm{g}$ of mitochondrial protein extracts from wild type (columns 2 and 3 ) or knockout (columns 4 and 5) MEFs. Mitochondrial proteins used in panels (c) and (e) were extracted from cells treated for $24 \mathrm{~h}$ with doxo $2 \mu \mathrm{M}$. Samples were resolved on SDS-PAGE gel, followed by autoradiography. (G) HtrA2 localization after doxo treatment. HtrA2 wild-type MEFs were treated with doxo $2 \mu \mathrm{M}$. Twenty-four hours later, samples were subjected to sub-cellular fractionation to analyze the protein of interest in each compartment. Twenty micrograms of each sample were resolved on SDS-PAGE gel, followed by immunoblotting. VDAC was used as a mitochondrial marker and actin as a cytoplasmic marker to ensure the absence of cross-contamination. Release of HtrA2 from mitochondria to cytoplasm was checked to confirm the results shown in panel (D). (H and I) p73 steady state in HtrA2 wild type and knockout MEFs. Twenty (H) and 200 (I) micrograms of whole-cell lysates from exponentially growing HtrA2 wild type and knock-out MEFs were resolved on SDS-PAGE gel, followed by immunoblotting. Actin was used as internal loading control

These results indicated that $\mathrm{HtrA} 2$ was important for bax induction. Furthermore, bax (Figure 5, box a), p21 (Figure 5, box b) mdm2 (Figure 5, box c) and vegf (Figure 5, box d) protein levels correlated with the activity of their respective promoters.

HtrA2 serine protease enhances p73 activity on bax. Previous results indicated a possible correlation between the HtrA2 and bax. To establish whether p73 might be one of the possible links, we performed a series of transient transfections in the p53-deficient $\mathrm{H} 1299$ cell line. Figure $6 \mathrm{a}$ shows that cotransfection of $\mathrm{HtrA} 2$ with p73 enhanced the production of bax mRNA compared with p73 transfection alone (compare columns 5 and 3). Transfection of the dominant-negative p73DD interfered with the bax mRNA induction. The contemporary presence of p73DD with p73 and HtrA2 blocked the bax upregulation (column 8) showed previously (column 5). Panel b of Figure 6 shows the expression of p21 mRNA under the same experimental conditions as in panel a. Cotransfection of p73 and HtrA2 (column 5) did not enhance the expression of p21 compared with p73 transfection alone. As a control of the HtrA2 activity, Figure $6 c$ shows the HtrA2-mediated cleavage of $p 73$. We also checked whether p73DD could be cleaved, and hence inactivated, by HtrA2. The amount of p73DD did not seem to decrease in the presence of $\mathrm{HtrA2}$, suggesting that the function of the dominant-negative form was preserved. In the same cellular system, regulation of bax and p21 at mRNA and protein levels after $\mathrm{HtrA} 2$ downregulation by siRNA was investigated. As expected, the downregulation of $\mathrm{HtrA} 2$ resulted in downregulation of bax at both mRNA and protein levels, whereas the expression of p21 was not modified (Figure 6d and e).

Endogenous HtrA2 enhances p73 activity on bax. The next step was to verify whether the endogenous HtrA2 could 

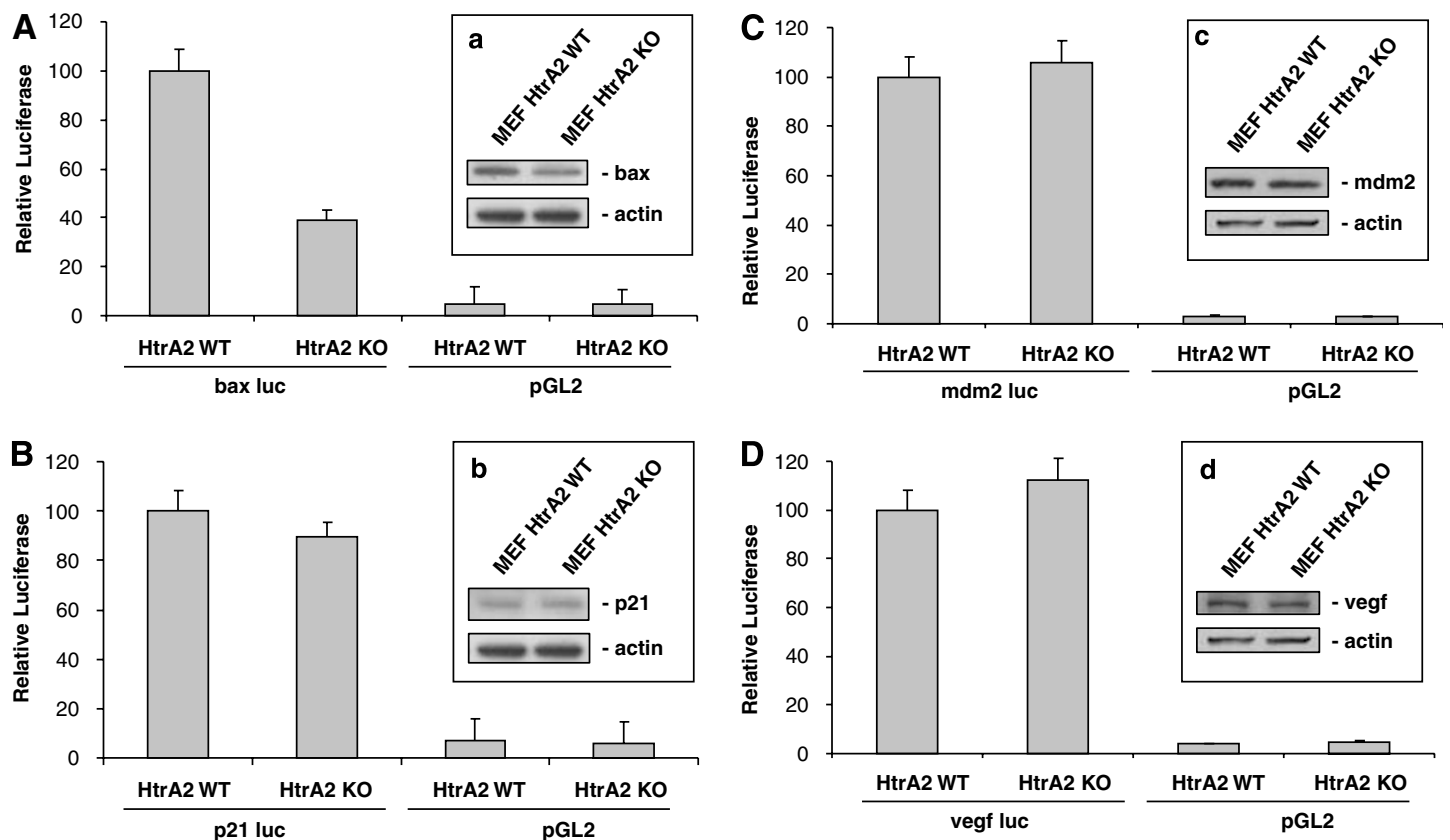

Figure 5 bax promoter basal activity is higher in presence of $\mathrm{HtrA2}$. Five hundred nanograms of plasmid encoding for a reporter gene (luciferase) driven by bax (BAX luc, (A)), p21 (p21 luc, (B)), mdm2 (mdm2 luc, (C)) or vegf (vegf luc, (D)) promoter were transfected into HtrA2 wild type and knockout MEFs. Luciferase activity was normalized by renilla activity. The average of three different experiments \pm S.D. is shown. Boxes a-d show the protein levels of bax, p21, mdm2 and vegf, respectively

enhance the activity of p73 on bax (Figure 7a). Transfection of p73 in HtrA2 wild-type MEFs resulted in an increase of bax mRNA detected by real-time PCR (column 2). Cotransfection of the dominant-negative p73DD with the transcriptionproficient p73 reduced this effect (column 4). In HtrA2knockout MEFs, bax transcription was not induced after p73 transfection (compare columns 6 and 2). In this cell line, the induction of bax transcription was comparable to the situation in which p73 was cotransfected together with the dominantnegative P73DD in HtrA2 wild-type MEFs (compare columns 6 and 4). Figure 7b shows how p21 was regulated in the same experimental conditions as for bax analysis. Transfection of $p 73$ in both cell lines, HtrA2 wild-type and knockout-knockout MEFs, resulted in the same p21 mRNA induction (compare columns 2 and 6), indicating that the presence or absence of HtrA2 had negligible effect. Cotransfection of the dominant-negative p73DD caused comparable reductions of $\mathrm{p} 21 \mathrm{mRNA}$ transcription in both HtrA2 wild-type and HtrA2-knockout MEFs (compare columns 4 and 8 ).

HtrA2 activity is necessary to stimulate p73-dependent apoptosis. The last step was to determine whether the increase of the apoptotic gene bax increased cell death. We investigated the activation of caspases 3 and 7 in a fluorimetry-based assay. Figure 7c reports the results: doxo treatment of $\mathrm{HtrA} 2$ wild-type MEFs for $6 \mathrm{~h}$ activated caspase 3 and 7 (column 3). Transfection of the dominant-negative p73DD $24 \mathrm{~h}$ before doxo treatment reduced caspase activity (compare columns 3 and 4).

As expected, the same experiment conducted in HtrA2knockout MEFs did not show any difference in caspase 3/7 activity when drug treatment alone was compared with doxo treatment plus p73DD transfection (compare columns 7 and 8). Caspase activity in HtrA2-knockout doxo-treated MEFs (both with or without p73DD) was comparable with the activity in $\mathrm{HtrA} 2$ wild-type MEFs treated with the drug and transfected with p73DD. These results support the idea that p73 can induce caspase activation and that $\mathrm{HtrA} 2$ activity is important for p73 activity (compare columns 7 and 8 with column 4).

We then checked whether caspase activation induced fragmentation of the nuclei, using DAPI/sulfurodamine B staining and microscope counting. The experimental conditions were the same as in the previous experiment, except that drug treatment was extended for $48 \mathrm{~h}$. Figure $7 \mathrm{~d}$ basically confirms that caspase activation showed in the previous experiment resulted in nuclei fragmentation and that the amount of activity was related to the amount of cell death. As previously shown for caspases, after drug treatment, apoptosis increased (column 3) more than when cells were transfected with p73DD before drug treatment (compare column 4 with column 3) in HtrA2 wild-type MEFs. Similarly, in HtrA2-knockout MEFs, the presence or the absence of the dominant-negative p73DD did not change the levels of apoptosis, which were comparable with the level in $\mathrm{HtrA} 2$ wildtype MEFs transfected with dominant-negative p73 before treatment (compare columns 7 and 8 with column 4).

\section{Discussion}

p73, a member of the p53 family, is a protein regulated by several complex mechanisms. ${ }^{2,21}$ The p73 gene contains two independent promoters that can regulate the expression of proteins with different behavior: TA promoter (P1) drives the expression of TA isoforms that induce cell-cycle arrest and apoptosis, whereas DN promoter (P2) controls the production 


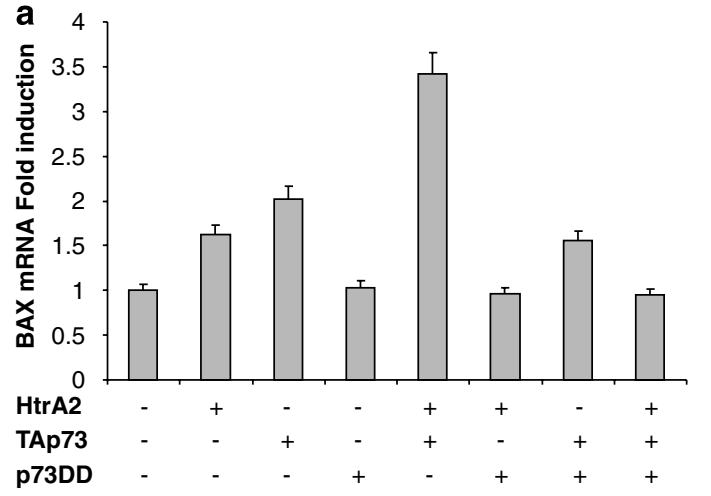

C

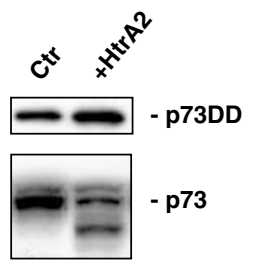

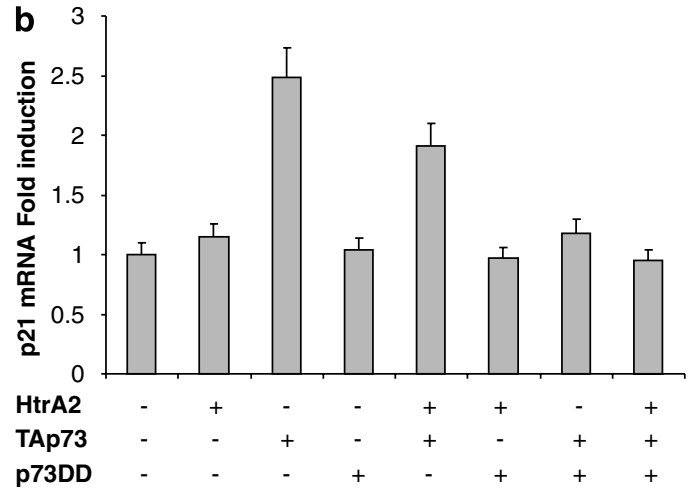

e d

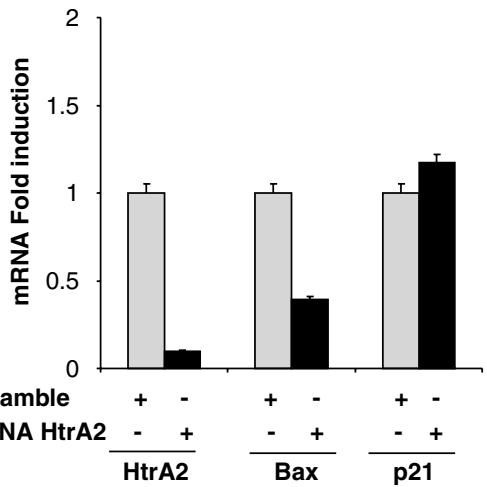

SiRNA HtrA2 - +

Scramble

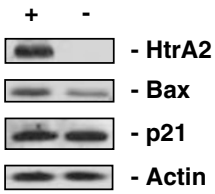

Figure 6 p73 regulates bax expression in presence of HtrA2. (a and b) H1299 cells seeded in six-well plates were transfected with the plasmid encoding for TAp73, HtrA2 and p73DD in the combinations shown. Twenty-four hours after transfection, RNA was extracted and bax (a) expression and p21 (b) were analyzed by Real Time RT-PCR using actin as a calibrator gene. The average of three different experiments \pm S.D. is shown. (c) H1299 cells seeded in six-well plates were cotransfected with the plasmid encoding for TAp73 or p73DD with wild-type HtrA2 in the conditions reported in panels (a) and (b). Twenty-four hours after transfection, $20 \mu \mathrm{g}$ of whole-cell lysates were resolved on SDS-PAGE gel, followed by immunoblotting. P73DD was digested to check for degradation and loss of function. (d and e) Downregulation of HtrA2 by siRNA influences bax mRNA and proteins levels: H1299 cells seeded in 24-well plates were transfected with equal amounts (35 pmoles) of scramble or HtrA2 siRNA. Forty-eight hours after transfection, mRNA and proteins were investigated by real-time PCR and western blot, respectively

of DN isoforms that have oncogenic features. ${ }^{7,21,22}$ In addition to the two promoters, the products of transcription can be spliced in a variety of isoforms (form $\alpha$ to $\zeta$ ) with different specificity and transcriptional activity. ${ }^{1,13,21}$ The $\alpha$-isoform contains an inhibitory domain in its C-terminal portion in fact, and the $\beta$-form that lacks this part of the protein is a more powerful transcription factor. ${ }^{21}$

We have characterized a new activation mechanism based on removal of the extreme C-terminal part of the p73 $\alpha$ isoform. This cleavage creates a new form of $p 73$ with peculiar characteristics. HtrA2, a member of the family of heat-shock endoproteases defined by bacterial HtrA, can cleave p73 $\alpha$ in the $\mathrm{C}$-terminal part of the protein. The other p73 isoforms $(\beta, \gamma$ and $\delta$ ) were not cleaved by $\mathrm{HtrA} 2$. HtrA2 targets many proteins such as IAPs, ${ }^{23} \mathrm{HAX} 1^{24}$ and ped/pea $15^{25}$ for their proteolysis. Here, for the first time, we have characterized a new role for HtrA2. The activity of HtrA2 is not necessary to eliminate a substrate, but it is needed to activate a new function of a substrate through elimination of one portion. In vitro and in vivo, HtrA2-cleaved p $73 \alpha$ in the C-terminal part. The proteolysis-inactive mutant was unable to cleave $p 73$, but did compete for the cleavage site on the target site on p73. In our hands, the cleavage of p73 was completely independent of caspase activation; in fact, inhibition of caspases activation with Z-vad-fmk pretreatment did not interfere with p73 cleavage in cells. Although HtrA2 is mainly localized in mitochondria, after stress we detected accumulation of $\mathrm{HtrA} 2$ in the nuclear compartment, where it can cleave p73. Colocalization of p73 and HtrA2 could be detected in the nucleus by confocal microscopy and by co-immunoprecipitation experiment. The finding that in two isogenic systems, the physiological levels of full-length $\mathrm{p} 73 \alpha$ were higher in HtrA2knockout MEFs than in wild-type MEFs seems to confirm that p73 is differently regulated in the two systems and that the HtrA2-mediated cleavage of p73 occurs in physiological conditions. In coherence with this, increasing amounts of proteins loaded into an SDS-PAGE indicated a lowermolecular-weight band detected by the anti-p73 antibody in an extract from HtrA2 wild-type MEFs. The cleavage of TAp73 $\alpha$ mediated by HtrA2 influences the activation of p73 downstream genes.

As a paradigm, we showed that HtrA2-cleaved p73 preferentially activated a proapoptotic gene such as bax, whereas the activation of a cell-cycle arrest-inducing gene, p21, was not affected. This in turn increased p73-dependent apoptosis. This mechanism, observed after cell damage, offers an additional strategy for the regulation of p73dependent apoptosis and might be used to modulate this activity in cancer cells after chemotherapy. Despite the effects on apoptosis seem to be quite specific, we also investigated the induction of cell-cycle-specific arrest and the appearance of a senescence-like phenotype without observing any effect 

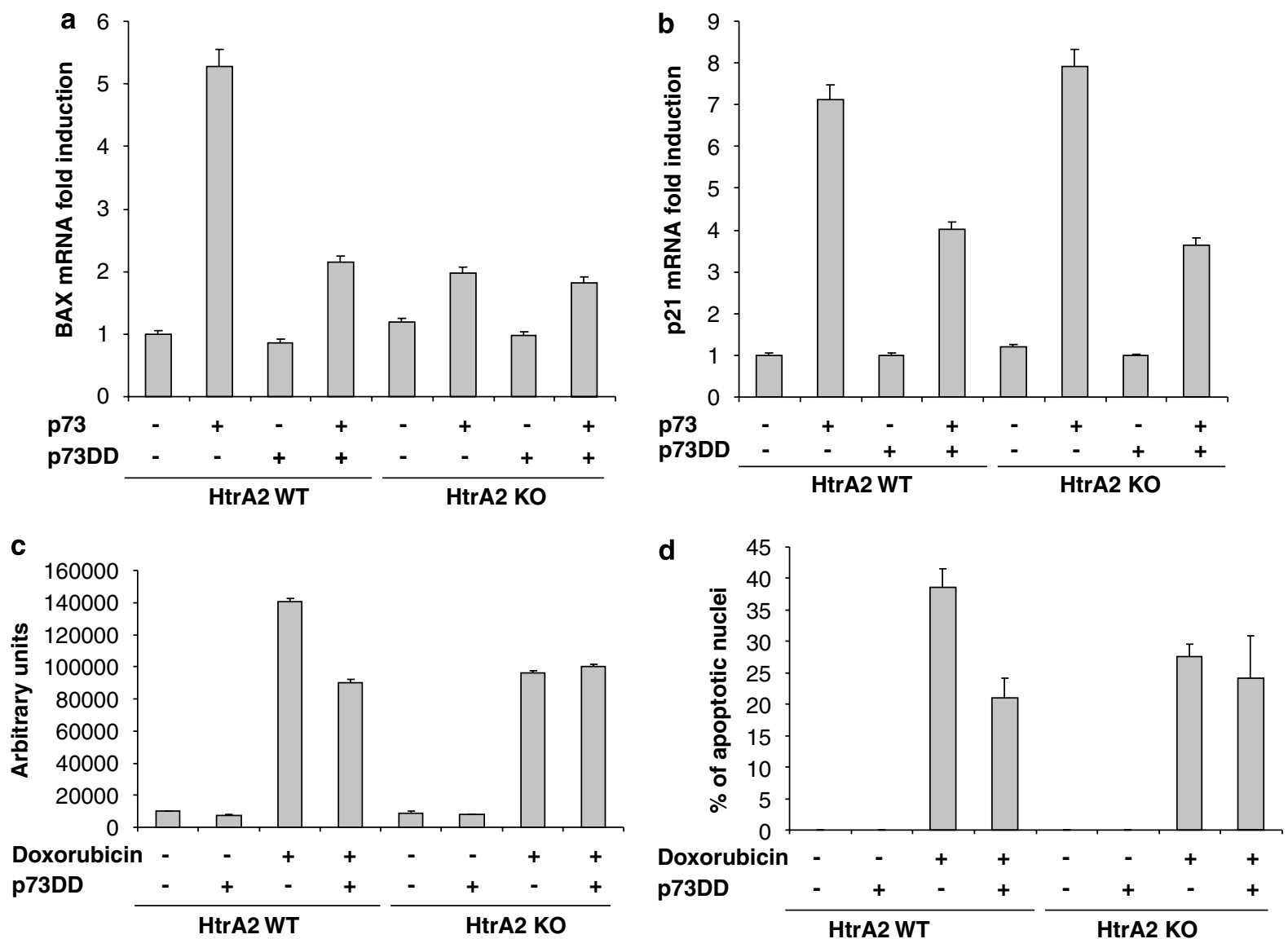

Figure 7 (a and $\mathbf{b})$ HtrA2 influences p73-mediated bax induction. Wild-type and knockout MEFs were transfected with TAp73 and p73DD encoding plasmid in the combinations shown. Twenty-four hours after transfection, RNA was extracted and real-time RT-PCR was performed to measure the levels of bax mRNA (a) and p21 (b). Actin was used as a calibrator gene. The average of three different experiments \pm S.D. is shown. (c) HtrA2 enhances the activity of p73 on caspase activation. Wild-type and knockout MEFs transfected with the P73DD-encoding plasmid or with the empty vector were seeded in 96-well plates. Twenty-four hours after transfection, cells were treated with doxo $1 \mu \mathrm{M}$ for $6 \mathrm{~h}$ and then caspase 3 and caspase 7 activities were assessed. The average of three different replicates \pm S.D. is shown. (d) p73 and HtrA2 cooperate to enhance apoptosis induction. Wild-type and knockout MEFs transfected with the p73DD-encoding plasmid or with the empty vector were seeded on a coverslip. Twenty-four hours after transfection, cells were treated with doxo $1 \mu \mathrm{M}$ for $48 \mathrm{~h}$ and then cells were stained and counted. The average of the percentage of apoptotic nuclei \pm S.D. is shown

(data not shown). Experiments to identify the precise site of cleavage of $\mathrm{HtrA} 2$ are in progress. Analysis of the sequence of the p73 C-terminus showed several potential cleavage sites. The literature, however, indicates that HtrA2 can cleave different sequences making any assumption, based on the peptide sequence, difficult. The reported evidence indicates that the cleavage involves removal of the inhibitory domain in the C-terminal portion of the p $73 \alpha$ isoform, which exerts general inhibitory activity on the protein's transcription capacity. ${ }^{21}$ The presence of HtrA2 and p73 in cells and possibly the formation of the cleaved form of p73 is important for caspase activation and apoptosis too. Without p73 or HtrA2, the levels of caspase activation and apoptosis levels were lower than in situations in which both proteins were present.

In conclusion, our findings indicate that the activity of TAp73 $\alpha$ on bax regulation needs to be stimulated through HtrA2 cleavage in the protein's C-terminal portion. The study also shed light on a novel activation mechanism of p73 that should be considered in cells that seem to show defects in apoptosis. Finally, it will be interesting to further analyze the role of $\mathrm{HtrA} 2$ in the cleavage of the $\mathrm{N}$-terminal truncated form of $p 73 \alpha$, which could have implications in the TAp73/DNp73/ p53 regulatory loop.

\section{Materials and Methods}

Cell cultures, transfections and drug treatments. The human nonsmall-cell lung carcinoma cell line $\mathrm{H} 1299$ was grown in RPMI1640 medium. Colon carcinoma cell line HCT116 was grown in Iscove's modified Dulbecco's medium. Immortalized MEFs (MEF HtrA2 $2^{+1+}$ and MEF HtrA2 $2^{-l-}$, kindly supplied by Dr Martins, Leicester, UK) were grown in Dulbecco's modified Eagle's medium. All media were supplemented with $10 \%(\mathrm{v} / \mathrm{v})$ fetal bovine serum and $2 \mathrm{mM}$ glutamine (Cambrex BioScience, Milan, Italy). Cells were maintained at $37^{\circ} \mathrm{C}$ in a humidified atmosphere of $5 \%(\mathrm{v} / \mathrm{v}) \mathrm{CO}_{2}$ in air.

Transient transfections were performed using Lipofectamine 2000 (Invitrogen, Italy) according to the manufacturer's protocol. Doxo (Sigma, Italy) was dissolved in medium just before use.

Plasmids. All p73 isoform expression plasmids (pCDNA3-TAp73 $\alpha$, pCDNA3TAp73 $\beta$, pCDNA3-TAp73 $\gamma$, pCDNA3-TAp73 $\delta$, pCDNA3-DNp73 $\alpha$, pCDNA3DNp73 $\beta$ ) were obtained from the laboratory of Dr V De Laurenzi (Rome). p73DD was obtained from $\mathrm{Dr} \mathrm{K}$ Sabapathy (Singapore). The pCMV/SPORT-HtrA2 expression plasmid was a kind gift from $\mathrm{Dr}$ LM Martins (Leicester). The proteolytically inactive mutant of $\mathrm{HtrA2}, \mathrm{HtrA2}(\mathrm{S} 306 \mathrm{~A})$, was obtained using 
QuikChange site-directed mutagenesis kit (Stratagene, Italy) according to the manufacturer's instructions. The following primers were used: $5^{\prime}$-GCTATTGATTT TGGAAACGCTGGAGGTCCCCTGGTTAACC- $3^{\prime}$ as forward primer and $5^{\prime}$-GGTTA ACCAGGGGACCTCCAGCGTTTCCAAAATCAATAGC- $3^{\prime}$ as reverse primer.

The presence of the desired mutation was confirmed by plasmid sequencing.

siRNAs. Typically, 35 pmoles of each duplex siRNA were transfected with Lipofectamine 2000 in 24-well plates. Oligonucleotides were from Sigma-Proligo (Italy) and had the following sequences:

p53 (sense): $5^{\prime}-$ r(GCAUGAACCGGAGGCCCAUUU)-3' p53 (antisense): $5^{\prime}$-r(AUGGGCCUCCGGUUCAUGCUU)--3' HtrA2 (sense)(1): $5^{\prime}$-r(AACGGCUCAGGAUUCGUGGUU)-3 HtrA2 (antisense)(1): $5^{\prime}$-r(CCACGAAUCCUGAGCCGUUUU)- $3^{\prime}$ HtrA2 (sense)(2): 5'-r(GGGGAGUUUGUUGUUGCCAUU)-3' HtrA2 (antisense)(2): $5^{\prime}$-r(UGGCAACAACAAACUCCCCUU)-3'

Western blotting analysis. Cell extracts were prepared by lysing cells for $30 \mathrm{~min}$ on ice in $50 \mathrm{mM}$ Tris- $\mathrm{HCl}, \mathrm{pH} 7.4,250 \mathrm{mM} \mathrm{NaCl}, 0.1 \%$ Nonidet NP- $40,5 \mathrm{mM}$ EDTA, $50 \mathrm{mM} \mathrm{NaF}$ in the presence of aprotinin, leupeptine and phenyl-methylsulfonyl-fluoride (PMSF) as protease inhibitors. Insoluble material was pelleted at $13000 \times \mathrm{g}$ for $10 \mathrm{~min}$ at $4^{\circ} \mathrm{C}$ and the protein concentration was determined using a BioRad assay kit (BioRad, Rome, Italy). A 20- $\mu \mathrm{g}$ weight of total cellular proteins was separated on SDS-PAGE and electrotransferred to PVDF membrane (Millipore, Italy). Antibodies were diluted as needed in 5\% non-fat dry milk in TBS-Tween 20 $0.05 \%$ (TBS-T). Immunoblotting was carried out with the following antibodies: antip73 rabbit polyclonal antibody (Oncogene Research), anti-HtrA2 rabbit polyclonal antibody (R\&D Systems), anti-actin goat polyclonal antibody (Santa Cruz Biotechnology), anti-cytochrome $c$ mouse monoclonal antibody (Santa Cruz Biotechnology), anti-HA monoclonal antibody (Sigma), anti-VDAC goat polyclonal antibody (Santa Cruz Biotechnology) and anti-laminin B rabbit polyclonal antibody (Santa Cruz Biotechnology). Antibody binding was revealed using peroxidase secondary conjugated antibodies (Santa Cruz Biotechnology) and visualized by enhanced chemiluminescence (ECL; Amersham, Milan, Italy).

Immunoprecipitation. $\mathrm{H} 1299$ cells $\left(4 \times 10^{6}\right)$ were transfected with $3 \mu \mathrm{g}$ of wild-type HtrA2 and $3 \mu \mathrm{g}$ of p73 or empty vector using Lipofectamine 2000 according to the manufacturer's instructions. Cells were harvested $24 \mathrm{~h}$ following transfection and lysed in $300 \mu$ of the lysis buffer used for western blotting. Lysates were centrifuged for $5 \mathrm{~min}$ at $13000 \times g$ at $4^{\circ} \mathrm{C}$. Supernatants were incubated overnight with $2 \mu \mathrm{g}$ of anti-p73 antibody and then for $2 \mathrm{~h}$ with $\mathrm{A} / \mathrm{G}$ agarose beads (Santa Cruz Biotechnology). Lysates with bound complexes were washed five times with RIPA buffer. Bound complexes were released by heating at $95^{\circ} \mathrm{C}$ for $5 \mathrm{~min}$ resolved on $10 \%$ SDS-PAGE and analyzed by western analysis with anti-HtrA2 antibodies.

Protein in vitro translations. Recombinant proteins were obtained with TNT-Coupled Reticulocyte Lysate Systems (Promega, Milan, Italy) according to the manufacturer's instructions. Radiolabelled proteins were obtained by adding ${ }^{35}$ S Imethionine to the reaction mix. Radiolabelled translation products were resolved on SDS-PAGE and visualized by autoradiography.

Protein in vitro digestion. A $1-\mu$ l volume of recombinant $\mathrm{HtrA2}$ protein was added to $5 \mu \mathrm{l}$ of in vitro translation product and left to react for $2 \mathrm{~h}$ at $37^{\circ} \mathrm{C}$. The reaction was stopped by adding $10 \mu \mathrm{l}$ of protein loading buffer. Digestion products were resolved on SDS-PAGE and visualized by autoradiography.

Mitochondria and nuclei extraction. At least $5 \times 10^{6} \mathrm{H} 1299$ cells were scraped from the culture plates into ice-cold PBS and harvested by centrifugation at 1000 r.p.m. for $10 \mathrm{~min}$; the pellet was washed with PBS and resuspended in $700 \mu \mathrm{l}$ of ice-cold buffer A (250 mM sucrose; $20 \mathrm{mM}$ Hepes- $\mathrm{KOH}$, pH 7.5; $10 \mathrm{mM} \mathrm{KCl}$; $1.5 \mathrm{mM} \mathrm{MgCl} ; 1 \mathrm{mM} \mathrm{Na}-E D T A ; 1 \mathrm{mM} \mathrm{Na}-E G T A ; 1 \mathrm{mM} \mathrm{DTT} ; 0.1 \mathrm{mM}$ PMSF) and homogenized 20 times on ice with a Teflon potter to break open the cell membranes. The homogenate was centrifuged twice at $750 \times g$ for 10 min at $4^{\circ} \mathrm{C}$ to discard nuclei and whole cells. The supernatants was centrifuged at $10000 \times g$ for $15 \mathrm{~min}$ at $4^{\circ} \mathrm{C}$ to recover the pellet (mitochondria-containing fraction) and supernatant (cytosolic fraction). The mitochondrial pellet was resuspended in $100 \mu \mathrm{l}$ of buffer A. Protein expression in nuclei and soluble fraction was determined as previously described. ${ }^{26}$
Real-time PCR. A 200-ng weight of total RNA was retrotranscribed in $20 \mu$ of reaction mix with TaqMan Reverse Transcription kit (Applied Biosystems, Monza, Italy) according to the manufacturer's instructions, and $2 \mu$ of this mixture were amplified by real-time PCR. Real-time PCR was then used to quantify bax, p21 and $\mathrm{HtrA2}$, with actin as internal control. Primers and TaqMan probe were purchased as ready-to-use solutions (Assay on Demand, Applied Biosystems). Real-time PCR was performed using 7900HT Sequence Detection System (Applied Biosystems).

Luciferase assay. Cells seeded in six-well plates were transfected with $0.5 \mu \mathrm{g}$ of purified plasmid and $0.5 \mu \mathrm{g}$ of PRL-SV40 (Promega) as internal normalization control, using Lipofectamine 2000 (Invitrogen). Twenty-four hours after transfection, luciferase and reporter gene activities were evaluated using the Dual Luciferase system (Promega) according to the manufacturer's instructions.

Caspase 3 and caspase 7 activity assay. Twenty-four hours after transfection with the plasmids and Lipofectamine 2000, cells were seeded in 96-well plates. Twenty-four hours after plating, drug treatment was performed and $6 \mathrm{~h}$ later caspase activity was assessed using the caspase-Glo 3/7 Assay (Promega) according to the manufacturer's instructions.

Confocal microscopy. Cells grown on glass coverslips were treated with Doxo for $14 \mathrm{~h}$. Cells were then washed twice with cold PBS, fixed in $4.5 \%(\mathrm{w} / \mathrm{v})$ paraformaldehyde in PBS and permeabilized with $0.2 \%$ Triton X-100 in PBS for $5 \mathrm{~min}$. After permeabilization, cells were incubated for $30 \mathrm{~min}$ in $1 \%$ BSA-PBS and then incubated for $1 \mathrm{~h}$ with primary antibodies (1:1000 dilution), washed and then incubated with the fluorescently labelled secondary antibodies (Molecular Probes), counterstained with DAPI for 3 min and mounted. Confocal imaging was performed by using a 480-nm ion argon laser and a 542-nm helium/neon laser connected to a Zeiss Axiovert microscope with a $\times 63$ oil-immersion lens.

Apoptosis detected by nuclei staining. Twenty-four hours after transfection with plasmid and Lipofectamine 2000, cells were seeded on coverslips. Twenty-four hours after plating, drug treatment was performed and $48 \mathrm{~h}$ later cells were fixed and stained. Briefly, coverslips were washed with PBS and cells were fixed in $95 \%$ ethanol. Two hours later, coverslips were washed twice with PBS and twice with distilled water. Samples were then incubated at room temperature with DAPI/sulfurodamine B solution $(6 \mu \mathrm{g} / 100 \mathrm{ml} \mathrm{DAPI}, 1 \mathrm{mg} / 100 \mathrm{ml}$ sulfurodamina $B$ in Tris $/ \mathrm{HCl}, \mathrm{pH}$ 8). Twenty minutes later, coverslips were washed with distilled water and once dry mounted with Entellan (Sigma).

Acknowledgements. We thank $V$ Fedele for special advices on HtrA2 experiments. We thank V De Laurenzi, G Melino, K Sabapathy and LM Martins for reagents. JD Baggott kindly revised the paper. The generous contribution of the Italian Association for Cancer Research is gratefully acknowledged. This work was partially supported by grants from the Italian Ministry of Health, Fondazione CARIPLO and Negri-Weizmann to MB. FV is a visiting scientist from the Institute of Cytology, Russian Academy of Science, St Petersburg, Russia.

1. Moll UM, Erster S, Zaika A. p53, p63 and p73 - solos, alliances and feuds among family members. Biochim Biophys Acta 2001; 1552: 47-59.

2. Melino G, De Laurenzi V, Vousden KH. p73: friend or foe in tumorigenesis. Nat Rev Cancer 2002; 2: 605-615.

3. Zhu J, Jiang J, Zhou W, Chen X. The potential tumor suppressor p73 differentially regulates cellular p53 target genes. Cancer Res 1998; 58: 5061-5065.

4. Jost CA, Marin MC, Kaelin Jr WG. p73 is a simian [correction of human] p53-related protein that can induce apoptosis. Nature 1997; 389: 191-194.

5. Kaghad M, Bonnet $H$, Yang A, Creancier L, Biscan JC, Valent A et al. Monoallelically expressed gene related to $\mathrm{p} 53$ at $1 \mathrm{p} 36$, a region frequently deleted in neuroblastoma and other human cancers. Cell 1997; 90: 809-819.

6. Maisse $C$, Guerrieri P, Melino G. p73 and p63 protein stability: the way to regulate function? Biochem Pharmacol 2003; 66: 1555-1561.

7. Ishimoto O, Kawahara C, Enjo K, Obinata M, Nukiwa T, Ikawa S. Possible oncogenic potential of deltaNp73: a newly identified isoform of human p73. Cancer Res 2002; 62: 636-641.

8. Putzer BM, Tuve S, Tannapfel A, Stiewe T. Increased deltaN-p73 expression in tumors by upregulation of the E2F1-regulated, TA-promoter-derived deltaN'-p73 transcript. Cell Death Differ 2003; 10: 612-614.

9. Nakagawa T, Takahashi M, Ozaki T, Watanabe KK, Todo S, Mizuguchi $\mathrm{H}$ et al. Autoinhibitory regulation of $\mathrm{p} 73$ by delta Np73 to modulate cell survival and death through a 
p73-specific target element within the delta Np73 promoter. Mol Cell Biol 2002; 22: 2575 2585.

10. Zaika Al, Slade N, Erster SH, Sansome C, Joseph TW, Pearl M et al. DeltaNp73, a dominant-negative inhibitor of wild-type p53 and TAp73, is up-regulated in human tumors. J Exp Med 2002; 196: 765-780.

11. Vossio S, Palescandolo E, Pediconi N, Moretti F, Balsano C, Levrero M et al. DN-p73 is activated after DNA damage in a p53-dependent manner to regulate p53-induced cell cycle arrest. Oncogene 2002; 21: 3796-3803.

12. Grob TJ, Novak U, Maisse $C$, Barcaroli D, Luthi AU, Pirnia F et al. Human delta Np73 regulates a dominant negative feedback loop for TAp73 and p53. Cell Death Differ 2001; 8: 1213-1223.

13. De Laurenzi V, Costanzo A, Barcaroli D, Terrinoni A, Falco M, Annicchiarico-Petruzzelli M et al. Two new p73 splice variants, gamma and delta, with different transcriptional activity. J Exp Med 1998; 188: 1763-1768.

14. Concin N, Becker K, Slade N, Erster S, Mueller-Holzner E, Ulmer H et al. Transdominant deltaTAp73 isoforms are frequently up-regulated in ovarian cancer. Evidence for their role as epigenetic p53 inhibitors in vivo. Cancer Res 2004; 64: 2449-2460.

15. Slade N, Zaika Al, Erster S, Moll UM. DeltaNp73 stabilises TAp73 proteins but compromises their function due to inhibitory hetero-oligomer formation. Cell Death Differ 2004; 11: 357-360.

16. Hegde R, Srinivasula SM, Zhang Z, Wassell R, Mukattash R, Cilenti L et al. Identification of $\mathrm{Omi} / \mathrm{HtrA} 2$ as a mitochondrial apoptotic serine protease that disrupts inhibitor of apoptosis protein-caspase interaction. J Biol Chem 2002; 277: 432-438.

17. Suzuki $Y$, Imai $Y$, Nakayama H, Takahashi K, Takio K, Takahashi R. A serine protease, $\mathrm{HtrA2}$, is released from the mitochondria and interacts with XIAP, inducing cell death Mol Cell 2001; 8: 613-621.
18. Verhagen AM, Silke J, Ekert PG, Pakusch M, Kaufmann $\mathrm{H}$, Connolly LM et al. HtrA2 promotes cell death through its serine protease activity and its ability to antagonize inhibitor of apoptosis proteins. J Biol Chem 2002; 277: 445-454.

19. Jin S, Kalkum M, Overholtzer M, Stoffel A, Chait BT, Levine AJ. CIAP1 and the serine protease HTRA2 are involved in a novel p53-dependent apoptosis pathway in mammals. Genes Dev 2003; 17: 359-367.

20. Kuninaka S, lida SI, Hara T, Nomura M, Naoe H, Morisaki T et al. Serine protease Omi/HtrA2 targets WARTS kinase to control cell proliferation. Oncogene 2006; 26 2395-2406.

21. Marabese M, Vikhanskaya F, Broggini M. p73: a chiaroscuro gene in cancer. Eur J Cance 2007; 43: 1361-1372.

22. Zaika Al, Slade N, Erster SH, Sansome C, Joseph TW, Pearl M et al. DeltaNp73, a dominant-negative inhibitor of wild-type $\mathrm{p} 53$ and TAp73, is up-regulated in human tumors $J$ Exp Med 2002; 196: 765-780.

23. Yang QH, Church-Hajduk R, Ren J, Newild-typeon ML, Du C. Omi/HtrA2 catalytic cleavage of inhibitor of apoptosis (IAP) irreversibly inactivates IAPs and facilitates caspase activity in apoptosis. Genes Dev 2003; 17: 1487-1496.

24. Cilenti L, Soundarapandian MM, Kyriazis GA, Stratico V, Singh S, Gupta S et al. Regulation of HAX-1 anti-apoptotic protein by Omi/HtrA2 protease during cell death. $J$ Biol Chem 2004; 279: 50295-50301.

25. Trencia A, Fiory F, Maitan MA, Vito P, Barbagallo AP, Perfetti A et al. Omi/HtrA2 promotes cell death by binding and degrading the anti-apoptotic protein ped/pea-15. J Biol Chem 2004; 279: 46566-46572.

26. Marabese M, Vikhanskaya F, Rainelli $C$, Sakai T, Broggini M. DNA damage induces transcriptional activation of $\mathrm{p} 73$ by removing C-EBPalpha repression on E2F1. Nucleic Acids Res 2003; 31: 6624-6632.

Supplementary Information accompanies the paper on Cell Death and Differentiation website (http://www.nature.com/cdd) 